\title{
Identification of acetyltransferase genes (HAT1 and KAT8) regulating HBV replication by RNAi screening
}

\author{
Hui Wang ${ }^{1 *}{ }^{*}$, KeHui Liu ${ }^{1 \dagger}$, Bernard A. M. Fang ${ }^{2,3{ }^{\dagger}}$, HaiQing Wu ${ }^{1,4}$, FengDi Li ${ }^{1}$, XiaoGang Xiang ${ }^{1}$, WeiLiang Tang ${ }^{1}$,
} GangDe Zhao ${ }^{1}$, LanYi Lin', Shisan Bao ${ }^{1,2^{*}}$ and Qing Xie ${ }^{1 *}$

\begin{abstract}
Background: The initiation of hepatitis B virus (HBV) replication involves the formation of covalently closed circular DNA (cccDNA) and its transcription into pregenomic RNA (pgRNA) in hepatocyte nuclei. The regulatory mechanism of HBV replication by acetyltransferase is thus far not well understood, but human acetyltransferase has been reported as being involved in the regulation of HBV replication.

Results: Depletion of KAT8 or HAT1 via RNA interference (RNAi) markedly down-regulated HBV-DNA and pgRNA levels in HepG2.2.15 cells, with KAT8 knockdown reducing both HBsAg and HBeAg more than HAT1 knockdown. Consistent with these observations, HBV replication regulators hepatocyte nuclear factor-4-a (HNF4a) and peroxisome proliferator-activated receptor gamma coactivator- (PPARGC-) 1-a were decreased following knockdown of HAT1 or KAT8.
\end{abstract}

Conclusions: These data suggest that KAT8 or HAT1 regulate HBV replication and may be potential drug targets of anti-HBV therapy.

Keywords: KAT8, HAT1, HBV replication, pgRNA, RNAi screening

\section{Background}

Chronic hepatitis B virus (HBV) infection is a major global health issue, with over 350 million infected patients, who are subsequently susceptible to development of cirrhosis and hepatocellular carcinoma (HCC) worldwide [1, 2]. HBV is a noncytopathic virus with a 3.2-kb, partially double-stranded, relaxed circular (RC) DNA genome [3]. Following HBV infection, RC DNA translocates into nucleus, where it is repaired by cellular polymerase to form covalently closed circular DNA

\footnotetext{
*Correspondence: wanghuirj@163.com; bob.bao@sydney.edu.au; xieqingrjh@163.com

${ }^{\dagger}$ Hui Wang, KeHui Liu and Bernard A. M. Fang contributed equally to this work

1 Department of Infectious Diseases, Ruijin Hospital, School of Medicine, Shanghai Jiao Tong University, Shanghai, China

${ }^{2}$ Discipline of Pathology, School of Medical Sciences and The Bosch Institute, Charles Perkins Centre, The University of Sydney, Sydney, NSW 2006, Australia

Full list of author information is available at the end of the article
}

(cccDNA) that is subsequently transcribed into pre-core RNA, pgRNA, surface mRNA and X mRNA [4]. Viral RNAs are then transported to cytoplasm for translation into viral proteins (e.g., core proteins that are assembled to capsids). The newly synthesized viral genome can either be enveloped into mature viral capsids for secretion or re-enter the nucleus to replenish the cccDNA pool [3]. Residual HBV DNA reappears in serum and viral capsids later [5] but can be cleared to undetectable levels following treatment. In light of this, chronic HBV infection is thus largely attributed to persistence and replication of HBV cccDNA.

Nuclear HBV cccDNA is organized into a mini-chromosome by both histone and nonhistone proteins and is regulated in a manner similar to cellular chromatin [6]. A modification of chromatin architecture is required to allow access of condensed genomic DNA by the regulatory transcription machinery protein. Covalent histone modifications can be performed by enzymes such 
as histone acetyltransferases (HATs) [7]. Furthermore, multiple studies have observed that inhibition of histone deacetylase (HDAC), trichostatin A (TSA), valproic acid (VPA) and nicotinamide (NAM) enhances HBV replication, suggesting that histone acetylation may promote HBV replication $[7,8]$. Taken together, this suggests that epigenetic regulation of cccDNA mini-chromosome is implicated in the early events of HBV replication [6, 7].

To investigate the acetyltransferases implicated in HBV replication, RNA interference screening was performed using a lentiviral shRNA library. By comparing the levels of hepatitis B surface antigen ( $\mathrm{HBsAg}$ ) and pgRNA, depletion of histone acetyltransferase 1 (HAT1) or lysine acetyltransferase 8 (KAT8) markedly repressed HBV replication. In particular, knockdown of $K A T 8$ by lentivirus-mediated shRNA resulted in decreased HBsAg and hepatitis B envelope antigen $(\mathrm{HBeAg})$ levels. In addition to this, knockdown of HAT1 or KAT8 down-regulated the expression of hepatocyte nuclear factor-4- $\alpha$ (HNF4A) and peroxisome proliferator-activated receptor gamma coactivator- (PPARGC-) $1-\alpha$ at the transcriptional level. These observations suggest that both HAT1 and KAT8 may regulate HBV replication.

\section{Results}

RNAi screening reveals acetyltransferases regulate HBV replication

HepG2.2.15, a stable cell line in which complete HBVDNA is integrated into the cell genome, can constitutively express HBV genes and release HBV capsids, as reflected by the high levels of HBsAg and $\mathrm{HBeAg}$ detected in conditioned culture supernatant [9]. In light of these characteristics, HepG2.2.15 is thus an ideal cell model to study $\mathrm{HBV}$ replication. As HBsAg is an abundant viral surface antigen of HBV and can be detected at an appropriate sensitivity in the sera of HBV carriers [10], HBsAg was used as the marker for the primary RNAi screen. KAT5 shRNA lentivirus served as a positive control, as it has been reported that KAT5 binds to HBV cccDNA and regulates $\mathrm{HBV}$ replication [7], whereas the lentiviral vector served as a negative control.

The HBsAg level in cell supernatant was measured by ELISA, and cell viability was analyzed by cell proliferation assay at $96 \mathrm{~h}$ after lentiviral infection. Via subsequent analysis of the ratio of HBsAg to the number of cells, RNAi depletions of ATF2, HAT1, CSRP2BP, and KAT8 decreased the level of HBV in the supernatant, with a similar pattern was observed in KAT5 (Fig. 1b). To further verify the roles of these target genes in HBV replication, we investigated the relative pgRNA level after their knockdown, using qRT-PCR, as pgRNA not only serves the template of HBV RC DNA but also encodes viral replication proteins (i.e., the polymerase $(\mathrm{P})$ and core protein $(\mathrm{C}))[11,12]$. We observed RNAi depletion of HAT1 or KAT8 resulted a

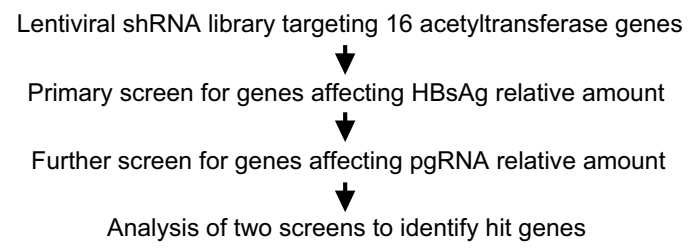

b
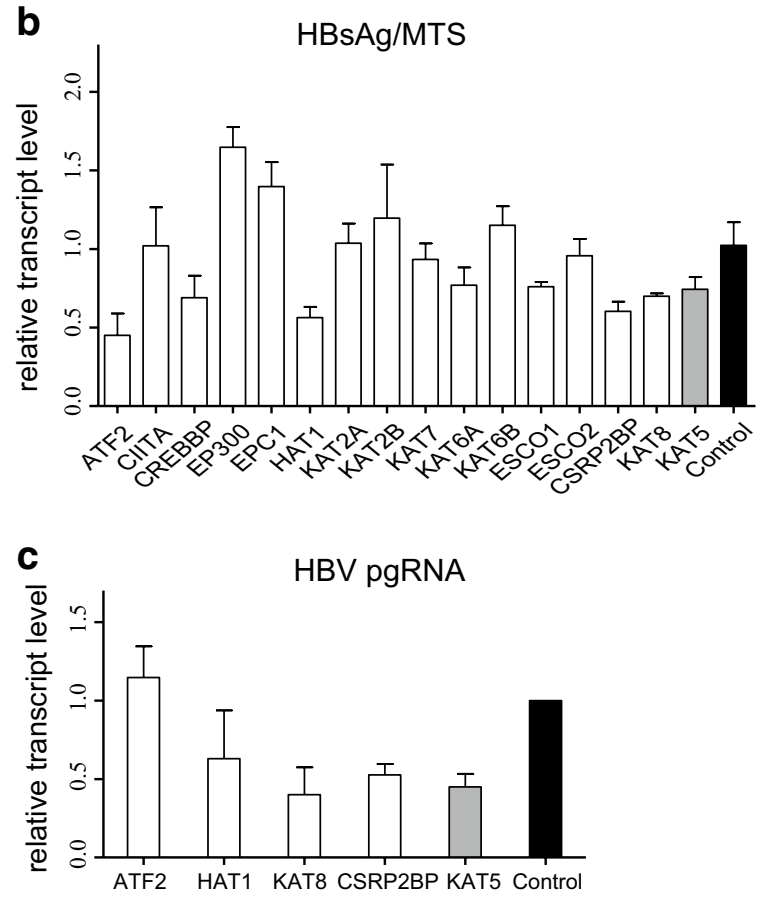

Fig. 1 RNAi screening reveals acetyltransferases regulate HBV replication. a Flowchart of RNAi screening. b The relative HBsAg after screen. HepG2.2.15 cells were infected with lentiviral shRNA library. HBsAg was measured by ELISA and cell growth was determined by MTS assay at $96 \mathrm{~h}$ after infection. The relative HBV level is given by the ratio of HBsAg/MTS. c Relative levels of pgRNA after screen. pgRNA was measured by qRT-PCR at $72 \mathrm{~h}$ after infection. The lentiviral vector served as a negative control and, KAT5 shRNA lentivirus served as a positive control, as it was reported that KAT5 bound to HBV cccDNA and regulate $\mathrm{HBV}$ replication

in a markedly decrease by $\sim 40$ and $\sim 60 \%$ in the level of pgRNA, which is consistent with the level observed in KAT5 [7], whereas ATF2 knockdown did not show this trend (Fig. 1c). Taken together, HAT1 and KAT8 are suggested to be involved in the regulation of HBV replication.

\section{HAT1 and KAT8 regulate HBV replication by suppressing the expression of $\mathrm{HBsAg}, \mathrm{HBeAg}$ and HBV-DNA}

The modulatory role of each shRNAs for both HAT1 and KAT8 in HBV replication was determined using a lentiviral vector as a carrier (Fig. 2a). HAT1 shRNA1 inhibited HBV replication by 0.64 (SD: 0.02) folds. HAT1 shRNA2 inhibited HBV replication by 0.59 (SD: 0.01) folds. HAT1 


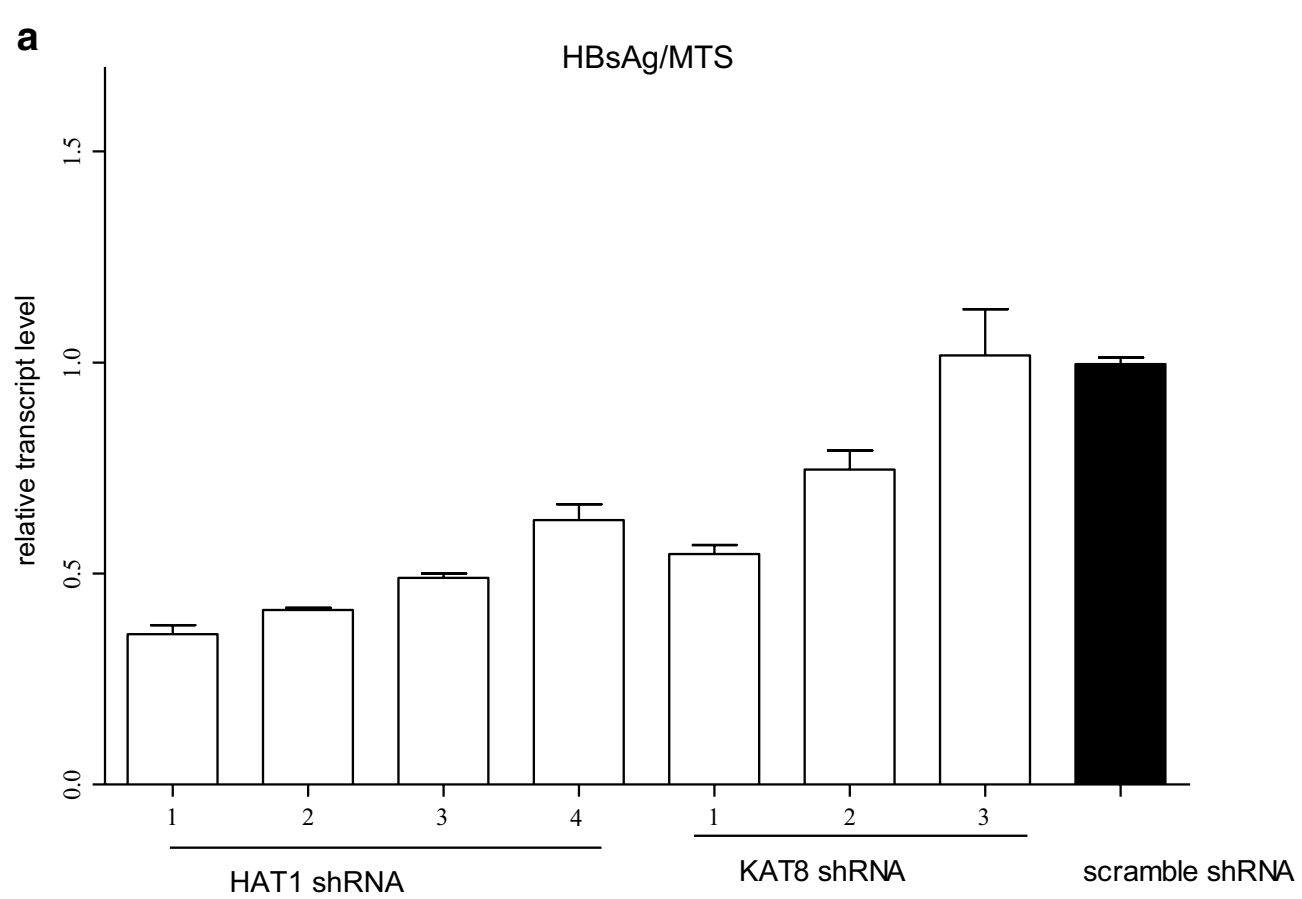

b

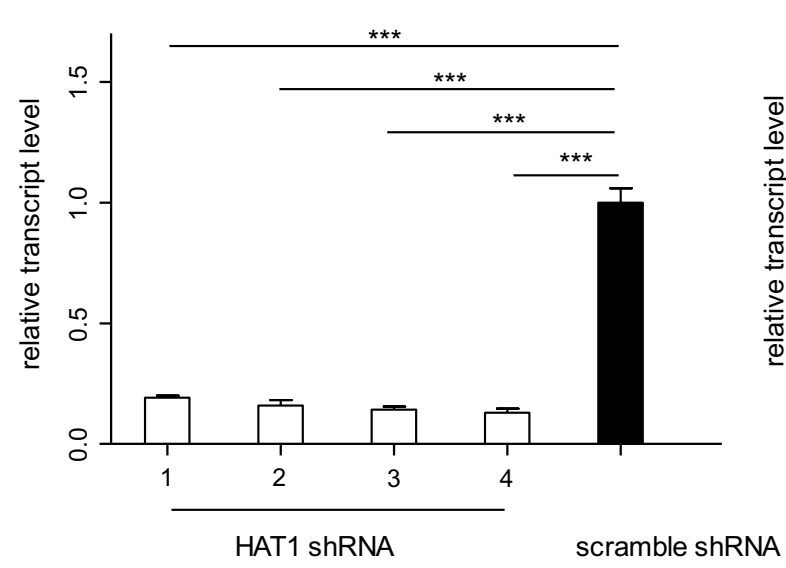

C

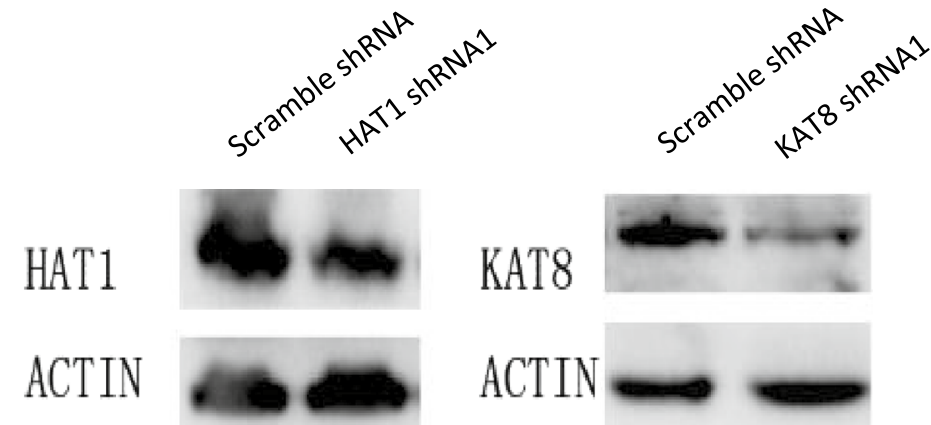

Fig. 2 The most inhibitory shRNAs of each of HAT1 or KAT8 in HepG2.2.5 cells. a Relative levels of HBsAg in HepG2.2.15 cells at $96 \mathrm{~h}$ after infection with lentiviral shRNAs of hit gene HAT1 and KAT8. The lentiviral vector served as a negative control. b Relative levels of HAT1 and KAT8 mRNA in HepG2.2.15 cells at $72 \mathrm{~h}$ after infection with shRNA lentivirus. Scrambled shRNA served as a negative control. $\left({ }^{*} P<0.05,{ }^{* *} P<0.001\right)$. c Protein level of HAT1 and KAT8 in HepG2.2.15 cells after infection with most inhibitory lentiviral shRNAs. $\beta$-actin served as an internal control 
shRNA3 inhibited HBV replication by 0.51 (SD: 0.01) folds. HAT1 shRNA4 inhibited HBV replication by 0.37 (SD: 0.04) folds. KAT8 shRNA1 inhibited HBV replication by 0.45 (SD: 0.02) folds. KAT8 shRNA2 inhibited HBV replication by 0.25 (SD: 0.05) folds. KAT8 shRNA3 promoted HBV replication by 0.02 (SD: 0.11) folds but this observation was not statistically significant $(P>0.05)$. Other than KAT8 shRNA3, all of the lentiviral shRNAs had a statistically significant inhibitory effect on HBV replication.

Furthermore, we also detected the inhibition effect of each shRNAs for both HAT1 and KAT8. It turned out that each four shRNAs for HAT1 effectively reduced HAT1 expression level $(P<0.001$ for all). For shRNA1 and shRNA2 of KAT8, there was a significant knockdown effect $(P<0.01$, respectively). KAT8 shRNA3 restrained KAT8 replication by 0.29 (SD: 0.07) folds and this observation also had a statistically significant inhibition $(P<0.05)$. The result above was shown that each shRNAs for KAT8 effectively reduced KAT8 levels. However, only 2 of them resulted in a lower of HBsAg whereas the third one had no effect. As a result, it might indicate that inhibition of KAT8 may not have an impact on HBsAg level.

After that, we tested the strongly inhibitory shRNA of each of HAT1 or KAT8 in HepG2.2.5 cells, HAT1 shRNA1 and KAT8 shRNA1, respectively. There was strong evidence suggesting that both HAT1 shRNA1 and $K A T 8$ shRNA1 had a significant knockdown effect $(P<0.001$ and $P<0.01$, respectively), as observed via qRT-PCR and Western blot (Fig. 2b, c).

The levels of HBsAg, HBeAg, and HBV-DNA at 72 and $96 \mathrm{~h}$ after shRNA transfection were determined. The expression of HBsAg was suppressed in these three samples at 72 or $96 \mathrm{~h}$, compared to control following HAT1 or KAT8 shRNA treatment (Fig. 3a). HBeAg was suppressed following KAT8 shRNA treatment only (Fig. 3b). As the duration of RNAi treatment progressed, the suppression of $\mathrm{HBsAg}$ or $\mathrm{HBeAg}$ expression was enhanced in all three samples after KAT8 shRNA treatment (both $P<0.0001$ ). In contrast, up-regulation of HBsAg or HBeAg was observed between 72 and $96 \mathrm{~h}$ after HAT1 shRNA treatment (both $P<0.0001$ ). Furthermore, HBV-DNA was also inhibited by HAT1 or KAT8 shRNAs treatment (Fig. 3c), but less inhibition of HBV-DNA was observed at $96 \mathrm{~h}$, compared to at $72 \mathrm{~h}$, after $H A T 1$ or KAT8 shRNA treatment (both $P<0.0001$ ). Moreover, the levels of $\mathrm{HBsAg}, \mathrm{HBeAg}$, and HBV-DNA were determined at 72 and $96 \mathrm{~h}$ after HAT1 inhibitor. The expression of HBsAg (Fig. 4a) and HBeAg (Fig. 4b) were suppressed by inhibitor $7476(P<0.001)$ or 7641 $(P<0.001)$ at both 72 and $96 \mathrm{~h}$ compared to control. No significant difference of HBV-DNA was observed following such treatments (Fig. 4c). There was significant
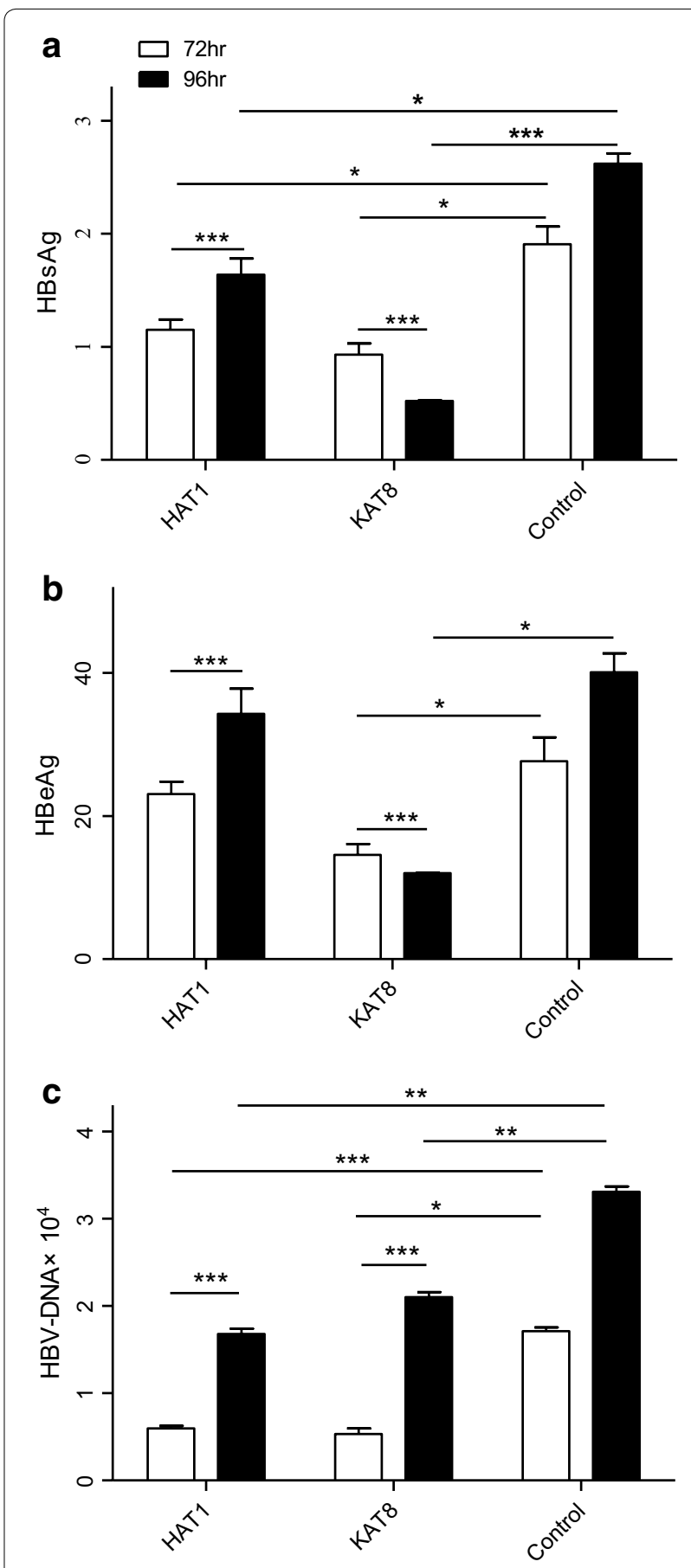

Fig. 3 HAT1 and KAT8 regulate HBV replication by suppressing the expression of HBsAg, HBeAg and HBV-DNA. a Relative levels of HBsAg at 72 and $96 \mathrm{~h}$ after infection with lentiviral shRNAs. b Relative levels of HBeAg at 72 and $96 \mathrm{~h}$ after infection with lentiviral shRNAs. c Relative levels of HBV-DNA at 72 and $96 \mathrm{~h}$ after infection with lentiviral shRNAs. $\left({ }^{*} P<0.05,{ }^{* *} P<0.001,{ }^{* * *} P<0.0001\right)$

suppression of HAT1 following inhibitor $7476(P<0.05)$ or $7641(P<0.0001)$ (Fig. $4 \mathrm{~d})$. It was demonstrated that the effects of HAT1 shRNAs and HAT1 inhibitors were 
similar but not exactly the same. The inhibitor potently reduced HBsAg throughout the $96 \mathrm{~h}$ trial but not the same as HAT1 shRNAs (Figs. 3a, 4a), and HBV-DNA was inhibited by HAT1 shRNAs treatment (Fig. 4b) but there was no significant difference of HBV-DNA following inhibitor treatment (Fig. 3c).

\section{HAT1 and KAT8 regulate the transcription level of HNF4A and PPARGC}

To investigate the mechanism by which HAT1 or KAT8 affected HBV replication, the expressions of HBV replication-related transcription factors Foxa1, Foxa2, Foxa3, CEBP, HNF1A, HNF1B, HNF4A, PPAR, FXR, FTF, and $P P A R G C$ were detected using qRT-PCR analysis.

The transcription levels of HNF4A were found to be suppressed by 0.44 (SD: 0.02) folds and 0.75 (0.04) folds in HepG2.2.15 cells infected with HAT1 shRNA and KAT8 shRNA, respectively (Fig. 5). The transcription levels of PPARGC were found to be suppressed by 0.25 (SD: 0.13) folds and 0.64 (SD: 0.02) folds in HepG2.2.15 cells infected with HAT1 shRNA and KAT8 shRNA, respectively (Fig. 5). This suggests that the acetyltransferases (HAT1 or KAT8) might regulate HBV replication via the transcription factor HNF4A and PPARGC. Further, this suggests that HNF4A and PPARGC may also be important in HBV regulation by HATs.

\section{Discussion}

HBV replication persists in chronic hepatitis B (CHB) patients, in whom there is an increased risk of developing heptocellular carcinoma (HCC) [13]. Current treatments for CHB include pegylated interferon alfa (PEG-IFNQ) and nucleoside or nucleotide analogues (NUCs) [14, 15]. However, PEG-IFN $\alpha$ therapy presents marked side effects [15] and $\sim 40 \%$ sustained virologic response [16]. Furthermore, NUCs drugs cannot completely clear HBV cccDNA from the hepatocyte and HBV mutants appear to be resistant to these drugs during therapy [17]. It is thus necessary to identify alternate targets for novel therapies to suppress HBV replication and to clear HBV [14, 18-20]. The formation of replicative intermediate cccDNA in the nucleus of infected hepatocytes, a process regulated by acetylated histones, is a key event in HBV replication [6, 8]. We thus hypothesize that HATs tightly regulate HBV replication and performed RNAi screening to proof this hypothesis.

The present study showed that HBV replication was regulated tightly by HAT1 and/or KAT8. However, the effects of HAT1 shRNAs and HAT1 inhibitors were not exactly the same, such different effects may be due to different inhibitions at mRNA/protein level by HAT1 shRNAs and HAT1, which will be further clarified in future. Moreover, $\mathrm{HBsAg}, \mathrm{HBeAg}$ and $\mathrm{HBV}$-DNA were observed as being markedly reduced in HepG2.2.15 cells when $H A T 1$ or $K A T 8$ were knocked down. Interestingly, there was minimal up-regulation of HBV-DNA between 72 and $96 \mathrm{~h}$ when HAT1 or KAT8 was knocked down. In contrast, there was down-regulation of HBsAg with time extension when $K A T 8$ was knocked down. Indeed, there are inconsistent reports about the association between HBV-DNA and liver histology in the different statuses of $\mathrm{HBeAg}$ patients [21]. Moreover, a recent study revealed that large number of virions without HBV-DNA was existed [22], suggesting that HBsAg and/or HBeAg levels probably do not reflect true HBV-DNA level but are instead reflective of the amount of HBV replication. From our qRT-PCR array, we identified a number of HBVreplication-related regulators that were up-regulated by knockdown of each target gene, such as HNF1A [23] or $H N F 1 B$, as well as other transcription factors for HBV replication that were down-regulated, such as PPARGC. These HBV-replication-related factors [24, 25] may preferentially regulate viral RNA synthesis, viral protein translation or viral DNA synthesis during virus lifecycle, thereby leading to different dynamics of $\mathrm{HBsAg}, \mathrm{HBeAg}$ and HBV-DNA.

It has been demonstrated that HAT1 plays an important role in controlling inflammatory NF- $\mathrm{KB}$ at the transcriptional level by acetylation of the transcriptional regulator PLZF [26]. However, the differential regulation of HAT1 on HBV-replication-related transcription factors remains to be investigated. HNF4A has been demonstrated to promote HBV replication via pgRNA in hepatic and non-hepatic cells [27]. HNF4A has been observed as being post-transcriptionally reduced by analogues based on the non-nucleoside natural product helioxanthin, suggesting it as a potential therapeutic target in the management of HBV [28]. In our study, we found that HAT1 or KAT8 all modulate HNF4A transcription. The peroxisome proliferator-activated receptors (PPARs), namely PPAR- $\alpha$, PPAR- $\beta$, and PPAR- $\gamma$, are ligand-activated transcription factors of the nuclear hormone receptor superfamily [24, 25]. These receptors act as lipid metabolism regulators and play key roles in cellular proliferation, differentiation, and apoptosis $[29,30]$. In this present study, PPARGC may be demonstrated to be another HBV transcription modulator that is regulated by HAT1 and KAT8. PPARGC synergizes with HNF4A to activate downstream genes transcription and HBV replication [31]. These findings suggest that HNF4A and PPARGC may be critical intermediate modulators that mediate regulation of $\mathrm{HBV}$ replication by histone acetyltransferases. In addition to this, HAT1 and KAT8 were also observed to differentially modulate expression of $\mathrm{HBV}$ transcription regulators, suggesting that they may regulate HBV in different manners. 


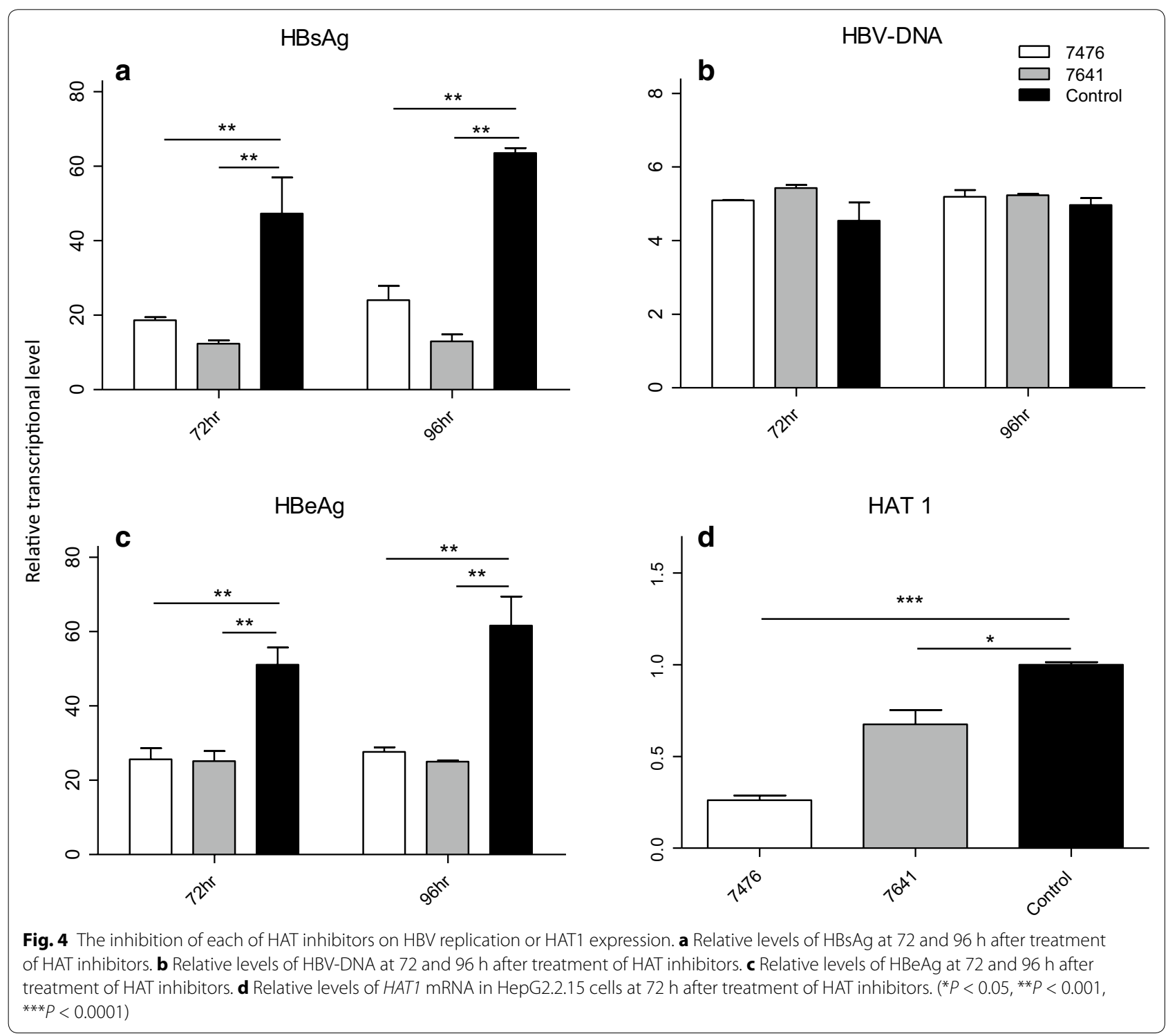

A more complete understanding of the underlying mechanisms involved in the interaction between HBV and its host factors will be valuable in the identification of new cellular drug targets. The potential use of the novel antiviral targets to inhibit viral replication via interfering virus-host interaction, may have some advantages, such as fewer side effects compared to direct antiviral therapies. In addition, novel therapeutics with a broader spectrum of activity against the various HBV genotypes are necessary to overcome the vast genetic heterogeneity and antiviral drug resistance [32].

\section{Conclusions}

The present study showed that HAT1 and KAT8 (human acetyltransferases) play an important role in regulating
HBV replication, indicating a potential underlying mechanism by which acetyltransferases influence HBV replication. Our observations may provide novel insights for identifying novel therapeutic targets for treating hepatitis B.

\section{Methods}

\section{Cell lines and culture}

The HBV-replication-stable HepG2.2.15 cell line was maintained in our laboratory, as previously described [9]. HEK293T cells were obtained from the American Type Culture Collection. HepG2.2.15 and HEK293T cells were maintained at $37^{\circ} \mathrm{C}$ in DMEM medium, supplemented with $10 \%$ fetal bovine serum (Life Technologies, Carlsbad, CA, USA), in a humidified atmosphere containing 


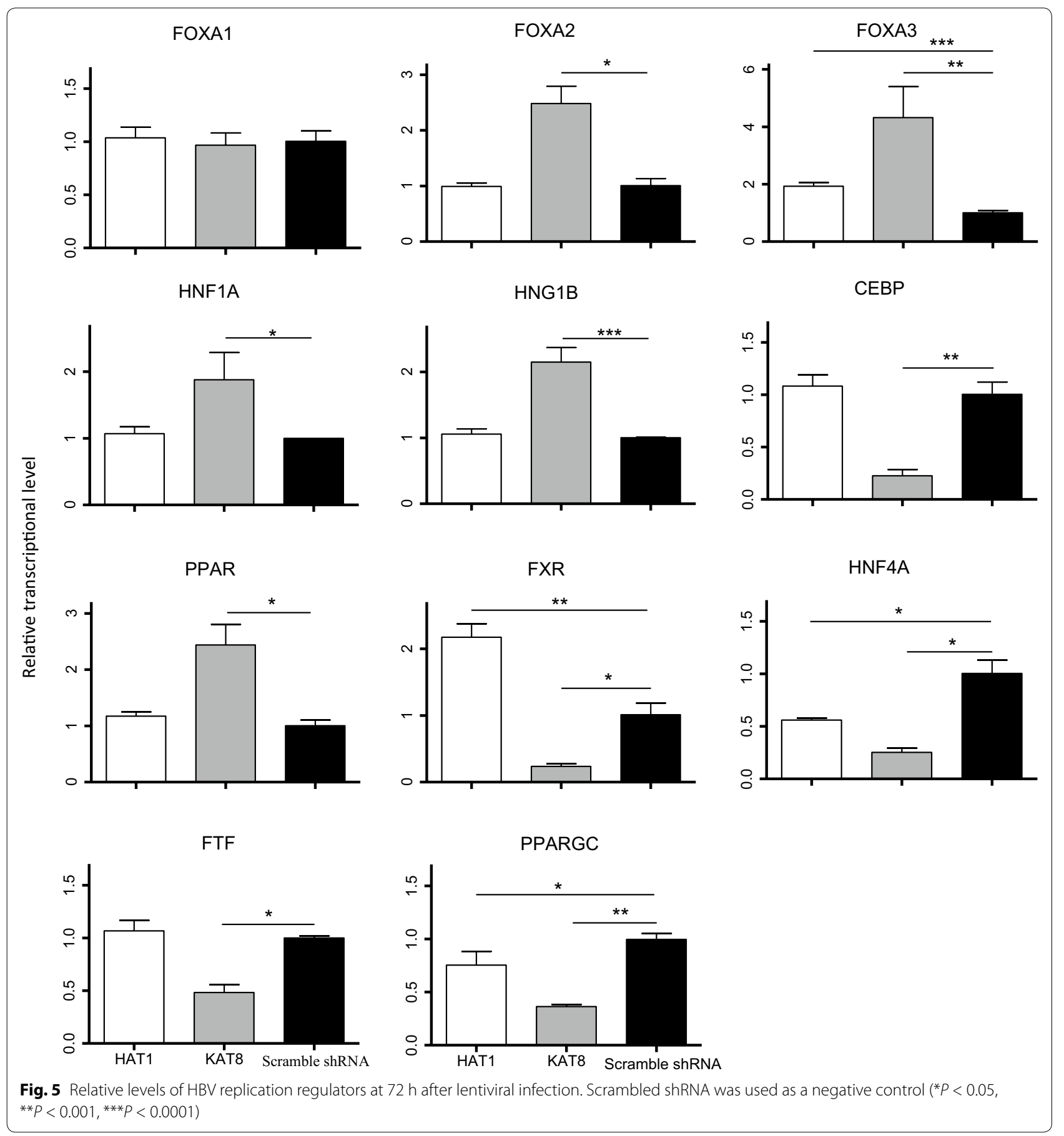

$5 \% \mathrm{CO}_{2}$. G418 (Life Technologies, Carlsbad, CA, USA) was also added to the medium to a final concentration of $380 \mu \mathrm{g} / \mathrm{mL}$ to maintain the HepG2.2.15 cells [9]. The details of HAT1 inhibitors were given by the site: http:// www.selleck.cn/search.html?searchDTO.searchParam = HAT+inhibitor\&sp=HAT\%252520inhibitor.

\section{Preparation of shRNA library and screen}

To identify which acetyltransferase genes are involved in HBV replication, RNAi screening was performed, as shown in Fig. 1a. RNAi screening has been widely used in the identification of host factors that regulate infection and replication by pathogens and 
viruses, including hepatitis C virus (HCV) [33]. shRNAs against 15 human acetyltransferase genes were designed, using an online tool available from the Broad Institute's RNAi Consortium (TRC) shRNA Library (http://www.broadinstitute.org/rnai/public/), a punchier lentiviral RNAi library that uses multiple distinct shRNA to knockdown most of the known human and mouse genes [34]. Four pairs of oligos were synthesized for each gene and cloned into modified FuGw lentiviral vector, harboring the U6 promoter for shRNA expression and tandem dimer Tomato gene (Tdtomato) for infection indication. (All of the oligos and scramble shRNA sequences are listed in Additionalfile 1: Table S1). All of the constructs were verified using colony PCR and using restriction enzyme digestion and subsequent co-transfection with packaging plasmids (Life Technologies, Carlsbad, CA, USA) in HEK293T cells.

HepG2.2.15 cells were infected with pooled lentiviral shRNA library in triplicate plates. The supernatant was collected 72 and $96 \mathrm{~h}$ after infection for Quantitative Real-time PCR (qRT-PCR) and HBsAg detection, respectively. RNA was extracted from the cultured cells using Trizol (Life Technologies, Carlsbad, CA, USA) for qRT-PCR. The forward and reverse primers for pgRNA are listed in Additional file 2: Table S2. Cellular RNA was extracted using Trizol (Life Technologies, Carlsbad, CA, USA) and cDNA was prepared from $1 \mu \mathrm{g}$ total RNA using the RevertAid ${ }^{\mathrm{TM}}$ Reverse Transcriptase kit (Thermo Fisher Scientific, Waltham, MA, USA). Amplification was performed as follows: $94{ }^{\circ} \mathrm{C}$ for $2 \mathrm{~min}$, then 40 cycles at $95{ }^{\circ} \mathrm{C}$ for $15 \mathrm{~s}, 6{ }^{\circ} \mathrm{C}$ for $30 \mathrm{~s}, 72{ }^{\circ} \mathrm{C}$ for $20 \mathrm{~s}$, and $72{ }^{\circ} \mathrm{C}$ for $20 \mathrm{~s}$. The primers for HAT1, KAT8 and other genes listed in Additional file 2: Table S2.

\section{Western blot}

The treated cells were lysated with RIPA buffer (Abcam, Cambridge, UK) and collected for western blot. Protein quantification was performed using BCA kit (Abcam, Cambridge, UK). Protein $(10 \mu \mathrm{g})$ was loaded for electrophoresis and transferred to PVDF membranes. The blots were blocked and labeled with primary antibodies (mouse anti-human HAT1, 1:2000 dilution; Santa Cruz Biotechnology, Dallas, TX, USA); rabbit anti-human KAT8, 1:2000 (Abcam, Cambridge, UK); or mouse antihuman $\beta$-actin 1:5000 (Santa Cruz Biotechnology, Dallas, TX, USA) overnight. Membranes were incubated with the secondary antibody (goat anti-rabbit-HRP or goat anti-mouse-HRP, 1:5000 dilution each; Amersham Pharmacia Biotech, Saclay, France). The signal was developed and imaged using ImageQuant ${ }^{\mathrm{TM}}$ LAS 4000 (Fujifilm, Tokyo, Japan).

\section{Enzyme-linked immunosorbent assay (ELISA)}

To detect the HBV expression in HepG2.2.15 cells, HBsAg and HBeAg levels in the medium at 72 and $96 \mathrm{~h}$ after lentiviral shRNA infection were semi-quantitatively determined using enzyme-linked immunosorbent assay (ELISA) kits (Abbott Diagnostics, Lake Forest, IL, USA) per the manufacturer's instructions. All experiments were performed in triplicate.

\section{HBV DNA quantification}

To detect the level of HBV replication in HepG2.2.15 cells, supernatant HBV DNA levels in the medium at 72 and $96 \mathrm{~h}$ after lentiviral shRNA infection were measured by the Applied Biosystems Real-Time PCR Prism 7500 System (Applied Biosystems, Carlsbad, CA, USA). All experiments were performed in triplicate.

\section{Cell proliferation assay}

Cell proliferation assay was performed in triplicate, using CellTiter $96^{\circledR} \mathrm{AQ}_{\text {ueous }}$ One Solution Cell Proliferation Assay kit (Promega, Madison, WI, USA) at $96 \mathrm{~h}$ after lentiviral shRNA infection, per the manufacturer's protocol.

\section{Statistical analysis}

The data are expressed as mean $\pm \mathrm{SD}$. One-way ANOVA and two-tailed Student's t-tests were used for Figs. 2, 3 and 4, using GraphPad Prism, Version 6.0 (GraphPad Software, San Diego, CA, USA). $P$-values $<0.05$ were considered statistically significant.

\section{Additional files}

Additional file 1: Table S1. Oligos and scramble shRNA sequences.

Additional file 2: Table S2. Primers for pgRNA and $q R T-P C R$.

\section{Abbreviations}

ccCDNA: covalently closed circular DNA; HAT: histone acetyltransferase; HBeAg: hepatitis $B$ envelop antigen; $H B S A g$ : hepatitis $B$ surface antigen; $H B V$ : hepatitis $B$ virus; HCC: hepatocellular carcinoma; HDAC: histone deacetylase; HNF4A: hepatocyte nuclear factor-4-a; PPAR: peroxisome proliferator-activated receptor; PPARGC-a: peroxisome proliferator-activated receptor gamma coactivator1-a; $R C$ : relaxed circular DNA.

\section{Authors' contributions}

$\mathrm{HW}, \mathrm{HQW}, \mathrm{SB}$, and QX designed the research. HW, KHL, BAMF, HQW, FDL, GDZ, and WLT conducted the research. HW and QX provided reagents and analytic tools. All authors prepared the figures and manuscript and read and approved the manuscript.

\section{Author details}

1 Department of Infectious Diseases, Ruijin Hospital, School of Medicine, Shanghai Jiao Tong University, Shanghai, China. ${ }^{2}$ Discipline of Pathology, School of Medical Sciences and The Bosch Institute, Charles Perkins Centre, The University of Sydney, Sydney, NSW 2006, Australia. ${ }^{3}$ Central Clinical School, Sydney Medical School, The University of Sydney, Sydney, NSW 2006, Australia. ${ }^{4}$ Department of Cardiology, Shanghai First People's Hospital, School of Medicine, Shanghai Jiao Tong University, Shanghai, China. 


\section{Acknowledgements}

This work was supported by grants from the National Natural Science Foundation of China (81070334), the National "Twelve-Five" Project of China (2012ZX10005004-002), the Committee of Science and Technology of Shanghai Municipal Government (13401902900), the Shanghai Public Health Outstanding Academic Leader Training Program (GWDTR201202), the Medical Key Project of Shanghai Municipal Health and Family Planning Commission (20134004), and the National Clinical Key Speciality Construction Project of China (Infectious Diseases).

\section{Competing interests}

The authors declare that they have no competing interests.

Received: 1 February 2015 Accepted: 23 November 2015 Published online: 04 December 2015

\section{References}

1. Dienstag JL. Hepatitis B virus infection. N Engl J Med. 2008;359(14):1486500. doi:10.1056/NEJMra0801644.

2. MacLachlan JH, Cowie BC. Hepatitis B virus epidemiology. Cold Spring Harbor Persp Med. 2015;. doi:10.1101/cshperspect.a021410.

3. Seeger C, Mason WS. Hepatitis B virus biology. Microbiol Mol Biol Rev MMBR. 2000;64(1):51-68.

4. Nassal M. HBV cccDNA: viral persistence reservoir and key obstacle for a cure of chronic hepatitis B. Gut. 2015; doi:10.1136/gutjnl-2015-309809.

5. Michalak TI, Pasquinelli C, Guilhot S, Chisari FV. Hepatitis B virus persistence after recovery from acute viral hepatitis. J Clin Investig. 1994:93(1):230-9. doi:10.1172/JCl116950.

6. Bock CT, Schwinn S, Locarnini S, Fyfe J, Manns MP, Trautwein C, et al. Structural organization of the hepatitis B virus minichromosome. J Mol Biol. 2001;307(1):183-96. doi:10.1006/jmbi.2000.4481.

7. Belloni L, Pollicino T, De Nicola F, Guerrieri F, Raffa G, Fanciulli M, et al. Nuclear $\mathrm{HBx}$ binds the HBV minichromosome and modifies the epigenetic regulation of cccDNA function. Proc Natl Acad Sci USA. 2009:106(47):19975-9. doi:10.1073/pnas.0908365106.

8. Pollicino T, Belloni L, Raffa G, Pediconi N, Squadrito G, Raimondo G, et al. Hepatitis $B$ virus replication is regulated by the acetylation status of hepatitis $\mathrm{B}$ virus cccDNA-bound $\mathrm{H} 3$ and $\mathrm{H} 4$ histones. Gastroenterology. 2006;130(3):823-37. doi:10.1053/j.gastro.2006.01.001.

9. Sells MA, Chen ML, Acs G. Production of hepatitis B virus particles in Hep $\mathrm{G} 2$ cells transfected with cloned hepatitis B virus DNA. Proc Natl Acad Sci USA. 1987;84(4):1005-9.

10. Kam W, Rall LB, Smuckler EA, Schmid R, Rutter WJ. Hepatitis B viral DNA in liver and serum of asymptomatic carriers. Proc Natl Acad Sci USA. 1982;79(23):7522-6.

11. Bartenschlager $\mathrm{R}$, Schaller H. Hepadnaviral assembly is initiated by polymerase binding to the encapsidation signal in the viral RNA genome. The EMBO J. 1992;11(9):3413-20.

12. Hirsch RC, Lavine JE, Chang LJ, Varmus HE, Ganem D. Polymerase gene products of hepatitis $B$ viruses are required for genomic RNA packaging as wel as for reverse transcription. Nature. 1990;344(6266):552-5. doi:10.1038/344552a0.

13. Yim HJ, Lok AS. Natural history of chronic hepatitis B virus infection: what we knew in 1981 and what we know in 2005. Hepatology. 2006;43(2 Suppl 1):S173-81. doi:10.1002/hep.20956.

14. Lo AO, Wong GL. Current developments in nucleoside/nucleotide analogues for hepatitis B. Exp Rev Gastroenterol Hepatol. 2014;8(6):607-22. doi:10.1586/17474124.2014.909724.

15. Tang CM, Yau TO, Yu J. Management of chronic hepatitis B infection: current treatment guidelines, challenges, and new developments. World J Gastroenterol WJG. 2014;20(20):6262-78. doi:10.3748/wjg.v20.i20.6262.

16. Lau GK, Piratvisuth T, Luo KX, Marcellin P, Thongsawat S, Cooksley G, et al. Peginterferon Alfa-2a, lamivudine, and the combination for HBeAg-positive chronic hepatitis B. N Engl J Med. 2005;352(26):2682-95. doi:10.1056/ NEJMoa043470.
17. Locarnini S. Primary resistance, multidrug resistance, and cross-resistance pathways in HBV as a consequence of treatment failure. Hep Intl. 2008;2(2):147-51. doi:10.1007/s12072-008-9048-3.

18. Lee $\mathrm{Cl}$, Kwon SY, Kim JH, Choe WH, Lee CH, Yoon EL, et al. Efficacy and safety of tenofovir-based rescue therapy for chronic hepatitis B patients with previous nucleo(s/t)ide treatment failure. Gut Liver. 2014;8(1):64-9. doi:10.5009/gnl.2014.8.1.64.

19. Ahmed M, Wang F, Levin A, Le C, Eltayebi Y, Houghton M, et al. Targeting the Achilles heel of the hepatitis B virus: a review of current treatments against covalently closed circular DNA. Drug Discovery Today. 2015;. doi:10.1016/j.drudis.2015.01.008

20. Seeger C, Mason WS. Molecular biology of hepatitis B virus infection. Virology. 2015;479:672-86. doi:10.1016/j.virol.2015.02.031.

21. Yuen MF, Ng IO, Fan ST, Yuan HJ, Wong DK, Yuen JC, et al. Significance of HBV DNA levels in liver histology of HBeAg and Anti-HBe positive patients with chronic hepatitis B. Am J Gastroenterol. 2004;99(10):2032-7. doi:10.1111/j.1572-0241.2004.40440.x.

22. Ning X, Nguyen D, Mentzer L, Adams C, Lee H, Ashley R, et al. Secretion of genome-free hepatitis $B$ virus-single strand blocking model for virion morphogenesis of para-retrovirus. PLoS Pathog. 2011;7(9):e1002255. doi:10.1371/journal.ppat.1002255.

23. Quasdorff M, Hosel M, Odenthal M, Zedler U, Bohne F, Gripon P, et al. A concerted action of HNF4alpha and HNF1alpha links hepatitis B virus replication to hepatocyte differentiation. Cell Microbiol. 2008;10(7):1478-90. doi:10.1111/j.1462-5822.2008.01141.x.

24. Curtil C, Enache LS, Radreau P, Dron AG, Scholtes C, Deloire A, et al. The metabolic sensors FXRalpha, PGC-1alpha, and SIRT1 cooperatively regulate hepatitis B virus transcription. FASEB J Off Publ Feder Am Soc Exp Biol. 2014;28(3):1454-63. doi:10.1096/fj.13-236372.

25. Dubuquoy L, Louvet A, Hollebecque A, Mathurin P, Dharancy S. Peroxisome proliferator-activated receptors in HBV-related infection. PPAR Res. 2009;2009:145124. doi:10.1155/2009/145124.

26. Sadler AJ, Suliman BA, Yu L, Yuan X, Wang D, Irving AT, et al. The acetyltransferase HAT1 moderates the NF-kappaB response by regulating the transcription factor PLZF. Nat Commun. 2015;6:6795. doi:10.1038/ ncomms7795

27. Li L, Oropeza CE, Sainz B Jr, Uprichard SL, Gonzalez FJ, McLachlan A. Developmental regulation of hepatitis B virus biosynthesis by hepatocyte nuclear factor 4alpha. PLoS One. 2009;4(5):e5489. doi:10.1371/journal. pone.0005489.

28. Ying C, Li Y, Leung CH, Robek MD, Cheng YC. Unique antiviral mechanism discovered in anti-hepatitis B virus research with a natural product analogue. Proc Natl Acad Sci USA. 2007;104(20):8526-31. doi:10.1073/ pnas.0609883104.

29. Michalik L, Auwerx J, Berger JP, Chatterjee VK, Glass CK, Gonzalez FJ, et al. International Union of Pharmacology. LXI. Peroxisome proliferator-activated receptors. Pharmacol Rev. 2006;58(4):726-41. doi:10.1124/pr.58.4.5.

30. Kersten S. Integrated physiology and systems biology of PPARalpha. Mol Metab. 2014;3(4):354-71. doi:10.1016/j.molmet.2014.02.002.

31. Shlomai A, Paran N, Shaul Y. PGC-1alpha controls hepatitis B virus through nutritional signals. Proc Natl Acad Sci USA. 2006;103(43):16003-8. doi:10.1073/pnas.0607837103.

32. de Chassey B, Meyniel-Schicklin L, Aublin-Gex A, Andre P, Lotteau V. Genetic screens for the control of influenza virus replication: from metaanalysis to drug discovery. Mol BioSyst. 2012;8(4):1297-303. doi:10.1039/ c2mb05416g.

33. Jayaswal S, Kamal MA, Dua R, Gupta S, Majumdar T, Das G, et al. Identification of host-dependent survival factors for intracellular Mycobacterium tuberculosis through an siRNA screen. PLoS Pathog. 2010;6(4):e1000839. doi:10.1371/journal.ppat.1000839.

34. Root DE, Hacohen N, Hahn WC, Lander ES, Sabatini DM. Genome-scale loss-of-function screening with a lentiviral RNAi library. Nat Methods. 2006;3(9):715-9. doi:10.1038/nmeth924. 